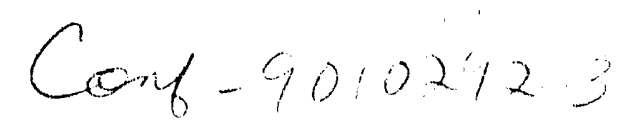

CONF-9010292--3

DE9 1006441

\title{
CHARACTERIZATION OF NANOCRYSTALLINE Pd BY X-RAY DIFFRACTION AND EXAFS*
}

\author{
J. A. Eastman ${ }^{1}$, M. R. Fitzsimmons ${ }^{2 * *}$, M. Müller-Stach ${ }^{2}$, G. Wallner ${ }^{2}$ and W. T. Elam ${ }^{3}$ \\ ${ }^{1}$ Materials Science Division, Argonne National Laboratory, Argonne, IL 60439 \\ 2Ludwig Maximilians-Universität, München, Germany \\ ${ }^{3}$ Naval Research Laboratory, Washington, DC 20375
}

December 1990

\section{DISCLAIMER}

\begin{abstract}
This report was prepared as an account of work sponsored by an agency of the United States Government. Neither the United States Government nor any agency thereof, nor any of their employees, makes any warranty, express or implied, or assumes any legal liability or responsibility for the accuracy, completeness, or usefulness of any information, apparatus, product, or process disclosed, or represents that its use would not infringe privately owned rights. Reference herein to any specific commercial product, process, or service by trade name, trademark, manufacturer, or otherwise does not necessarily constitute or imply its endorsement, recommendation, or favoring by the United States Government or any agency thereof. The views and opinicns of authors expressed herein do not necessarily state or reflect those of the United States Government or any agency thereof.
\end{abstract}

Submitted to a special issue of Acta Met. Proceedings of the Conference on Materials with Ultrafine Microstructures, Atlantic City, NJ, October 1-5, 1990.

*Work supported by the U. S. Department of Energy, BES-Materials Sciences, under Contract W/-31-109-Eng-38 (JE). Financial support was provided by the American-German Fulbright Comrnission (MF). Additional funding (MF, MM and GW) was provided by the German Federal Ministry for Research and Technology (BFMT) under contracts 523-4003-03M23 and 03PE1LMU/2.

**Current Address: Los Alamos Naticnal Laboratory, Los Alamos, NM 87545. 


\title{
CHARACTERIZATION OF NANOCRYSTALLINE Pd BY X-RAY DIFFRACTION AND EXAFS
}

\author{
J. A. Eastman ${ }^{1}$, M. R. Fitzsimmons ${ }^{2,}$, M. Müller-Stach², \\ G. Wallner ${ }^{2}$ and W. Tr. Elam ${ }^{3}$ \\ ${ }^{1}$ Argonne National Laboratory, Argonne, IL 60439 \\ 2Ludwig-Maximilians-Universităt, München, Germany \\ ${ }^{3}$ Naval Research Laboratory, Washington, D. C. 20375
}

\section{Intreduction}

Since the fraction of atoms located within a few atomic distances of one or more internal interfaces increases rapidly with decreasing grain size in the nanometer regime, it is expected that the structure of grain boundaries plays an important role in determining and controlling the properties of nanocrystalline materials. Studies characterizing the nature of the structure of grain boundaries in nanocrystalline materials have been controversial. Zhu, et al. [1] studied nanocrystalline $\mathrm{Fe}$ by $\mathrm{x}$-ray diffraction and concluded, based on a comparison of observed and calculated diffuse background intensities, that grain boundary regions in nanocrystalline materials lack both short- and long-range order. Haubold, et al. [2] concluded from an EXAFS investigation of nanocrystalline $\mathrm{Cu}$ and $\mathrm{Pd}$ that the boundary component of nanocrystalline materials represents a new solid-state structure with random atomic arrangement. In contrast to these conclusions, however, a high resolution electron microscopy study of nanocrystalline Pd found no evidence for extenjed, disordered boundary regions that would differ from boundaries in coarse-grained materials [3]. Recent results from $x$-ray diffraction [4], EXAFS [5] and hydriding studies [6] have yieldeo clear evidence that the boundaries in nanocrystalline Pd are ordered and/or extremely localized in nature. The present paper will summarize and discuss the principal results of these latter three studies.

\section{$X$-Bay Diffraction Studies at Ambient Temperature}

Nanocrystalline Pd was produced by the gas-condensation method [7] using the process first suggested by Gleiter [8]. In the present study $99.997 \%$ purity Pd wire was evaporated from a ceramic crucible in $500 \mathrm{~Pa}$ of $99.9999 \%$ $\mathrm{He}$ gas. The resulting ultrafine powder was collected and consolidated at arnbient temperature using a pressure of $\sim 1.4$ $\mathrm{GPa}$. Except during the evaporation of the $\mathrm{Pd}$, the entire process took place under vacuum conditions $\left(\sim 10^{-5} \mathrm{~Pa}\right)$ in order to ensure minimurn sample contamination. The sample was disk-shaped with a diameter of $9 \mathrm{~mm}$, a thickness of $0.18 \mathrm{~mm}$ and a density estimated at $80 \pm 10 \%$ of the literature value $\left(12.02 \mathrm{~g} / \mathrm{cm}^{3}\right.$ [9]). A coarse-grained reference foil cut to the same dimensions as the nanocrystalline sample was examined in order to obtain a direct comparison of the effect of grain size on diffracted interisity. The grain size of the nanocrystalline sample was estimated as 9 to $16 \mathrm{~nm}$, based on an analysis of $x$-ray Bragg peak broadening, while the coarse-grained sample had a grain size of several microns.

- Current Address: Los Alamos National Laboratory, Los Alamos, NM 87545 
Powder $x$-ray diffraction measurements were taken at the medium resolution two-axis spectrometer, $G 3$ HASYLAB, Germany, in reflection mode at ambient temperature. X-rays of wavelength $0.92 \AA$ were selected from the synchrotron ring. Scattered intensity was collected using a Ge energy dispersive counter with an energy window of $\sim 300$ $\mathrm{eV}$ width centered about the fundamental radiation of $13.48 \mathrm{keV}$. Intensity was recorded as a function of scattering angle $2 \theta$, and was normalized to the incident beam intensity. The position of the sample remained fixed during the experiment, and only the detector was scanned. Intensity measurements were corrected for absorption, Lorentz, polarization and atomic form factors. Corrected interssity data is shown in Figs. 1 (a) and 1 (b) for coarse-grained and nanocrystalline $P d$, respectively, as a function of scattering vector magnitude, $\tau=A \pi \sin \theta / \lambda$. Experimental intensity profiles were fit by a combination of a quadratic polynomial plus a Lorentzian function for each of the 16 observed Bragg peaks. Optimized parameters for the integrated intensity, the full-width at half-maximum intensity and the peak centroid position were obtained for each peak.

A quadratic was found to adequately represent the intensity not accounted for by the Lorentzian peaks and a direct comparison of optimized quadratics for both samples is shown in Fig. 1 (c). If atomic relaxations in grain boundary regions are large enough to produce significant amounts of broadly distributed diffuse scattering, then the intensity of the quadratic for the nanocrystalline sample should be significantly greater than that of the coarse-grained sample, since the coarse-grained sample contains a negligible total grain boundary volume. An increase of only $8 \pm 8 \%$ is observed in the region where $\tau>4 \AA^{-1}$, however. Larger differences are seen below this angle, but these differences are believed due to low intensity in this region and to artifacts of the fitting procedure. Zhu et al. [1] compared measured and computed interference functions for $\mathrm{Fe}$ and found that their model required large-magnitude random displacements of up to $50 \%$ of a nearest-neighbor distance in a four plane grain boundary region ( $50 \%$ of the total sample volume) in order produce enough background intensity to satisfactorily match that observed. The intensity between Bragg peaks was $~ 50 \%$ too low if smaller, but still significant, random displacements of up to $15 \%$ of a nearest-neighbor distance in the grain boundary region were used in their model (see Fig. 4 of [1]). An important limitation of the Zhu et al. study was that no direct experimental comparison was made of diffuse background intensities for nanocrystalline and coarse-grained samples. While it is possible that grain boundary structures in nanocrystalline Pd and Fe differ dramatically due to differences in crystal structure ( $f c c$ versus $b c c$ ) or due to the greater aftinity of Fe for boundary contaminants such as oxygen (the presence of which would produce diffuse intensity), our results demonstrate convincingly that random grain boundary relaxations lacking short-range order that would produce large broadly distributed diffuse background intensity do not occur in nanocrystalline Pd.

A significant difference between nanocrystalline and coarse-grained Pd was observed when comparisons were made of the two materials' Debye-Waller factors. The nanocrystalline Pd sample was found to have a significantly larger Debye-Waller factor than the literature value for $P d\left(0.56 \pm 0.04 \AA^{2}\right.$ versus $0.44 \pm 0.03 \AA^{2}$, respectively). This implies that the mean-squared displacements of atoms in the nanocrystalline Pd sample are $\sim 27 \%$ larger than in coarse-grained $P d$ at room temperature. Since the scattered intensity observed is averaged over the entire sample it is impossible to unambiguously determine the origin of these increased displacements. This behavior could result from differences in structure between grain boundaries and grain interiors (such as enhanced atom vibrations in the grain boundaries or ordered atomic relaxations in the boundaries that give rise to scattered intensity in the tails of the Lorentzian Bragg peaks) or from uniform displacements thiroughout the sample. The displacements may be of thermal and/or static origin and temperature measurements to discern between these two possibilities are currently in progress. 


\section{The Hydriding Behavior of Nanecrystalline Pd}

In situ $x$-ray diffraction studies of the hydriding behavior of nanocrystalline Pd were carried out at Argonne National Laboratory. A previous study by T. Mütschele and R. Kirchheim [10] found, based on the results of electrochemical hydrogen charging experiments, that the hydrogen solubility of nanocrystalline Pd differs from that of conventional, coarse-grained material. In particular they observed a narrowing of the miscibility gap in nanocrystalline $\mathrm{Pd}$ such that the maximum hydrogen solubility in the $\alpha-P d$ phase ( $\alpha$-max) approximately doubled while the minimum solubility in the $\beta-\mathrm{PdH}_{X}$ phase $(\beta-\mathrm{min})$ decreased by $\sim 25 \%$ at $333 \mathrm{~K}$. They attributed the increase in $\alpha-$ max to a larger solubility in the grain boundaries, while the decrease in $\beta$-min was proposed to be due to the presence of a disordered boundary region of thickness 0.7 to $1.1 \mathrm{~nm}$ that resisted transformation to the $\beta$-phase. Samples were produced for our study by the technique described above. Two nanocrystalline samples and one coarse-grained reference foil were examined. The two nanocrystalline samples had mean grain sizes of approximately 10 and $50 \mathrm{~nm}$, respectively. $X$-ray $\theta$ $2 \theta$ scans were obtained using $\mathrm{Cu} \mathrm{K \alpha}$ radiation $(\lambda=0.1542 \mathrm{~nm})$ while exposing the samples to controlled gaseous hydrogen pressures of 0 to $100 \mathrm{kPa}$ at ambient temperature. The smaller-grained nanocrystalline sample was cycled repeatedly through the $\alpha-\mathrm{Pd} \Leftrightarrow \beta-\mathrm{PdH} \mathrm{H}_{x}$ transformation, while the reference foil and larger-grained nanocrystalline samples were observed at several pressures, but were only subjected to one transformation cycle.

Lattice parameter changes as a function of hydrogen pressure were determined for all three samples and results for the $\alpha$-phase regime are shown in Fig. 2. The data clearly show that the change in lattice parameter at a given pressure depends on grain size, with the largest change occurring for the smallest-grained sample. Changes in lattice parameter can be directly related to changes in hydrogen concentration [11]. From lattice parameter measurements in the mixedphase regime it was determined that the $\alpha$-max concentration of the coarse-grained reference sample agrees well with the literature value at room temperature (H/Pd $\sim 0.01$ [12]), while the $\alpha$-max concentration of the smallest-grained nanocrystalline sample is approximately twice that, in agreement with the observations of Mütschele and Kirchheim [10]. Likewise, lattice parameter measurements of the $\beta$-phase in the mixed-phase regime showed that the $\beta$-min concentration decreased with decreasing grain size, indicating a narrowing of the miscibility gap as seen previously. A hydrogen pressure of $\sim 3 \mathrm{kPa}$ was required to cause the nanocrystalline samples to begin transforming to the $\beta$-phase, while a lower pressure of $\sim 2 \mathrm{kPa}$ caused the coarse-grained sample to begin transforming, indicating a change in the plateau pressure.

If the observed decrease in average $\beta$-min concentration is due to a significant fraction of the sample resisting transformation to the $\beta$-phase, this would be directly observable by $x$-ray diffraction. Since it is known from the background intensity comparisons described in the previous section that little additional broadly distributed diffuse scattering is produced in nanocrystalline Pd, one would expect to see a portion of the Pd Bragg peaks remaining even after prolonged hydrogen exposure above the plateau pressure. We found clear evidence that this is not the case for nanocrystalline Pd. Intensity data is shown in Fig. 3 for the smaller-grained nanocrystalline sample prior to hydrogen exposure (Fig.3(a)), in the mixed-phase regime (Fig.3(b)) and following complete transformation to the hydride phase (Fig.3(c)). This means again that there is no evidence for the presence of extended, disordered grain boundary regions in nanocrystalline $\mathrm{Pd}$, and that the observed narrowing of the miscibility gap must occur for another reason. An alternative explanation that we propose is that nanocrystalline Pd behaves as if it has a higher effective temperature than its coarsegrained counterpart. This is consistent with the observed increase in the Debye-Waller factor for nanocrystalline Pd, which corresponds to a decrease in Debye temperature of $\sim 60 \mathrm{~K}$. A change in effective temperature of this magnitude 
would be consistent with the observed change in solubility since $\alpha$-max for coarse-grained Pd is known to approximately double when the temperature is increased from 25 to $90^{\circ} \mathrm{C}[12]$.

\section{EXAFS Studies}

Haubold and co-workers [2] observed reductions in EXAFS amplitudes in nanocrystalline $\mathrm{Pd}$ and Cu compared to coarse-grained material. In order to verify and extend these results, we made EXAFS measurements on nanocrystalline Pd samples in both powdered and compacted forms, as well as on a Pd coarse-gritined reference foil. Samples were produced in the manner described above. Measurernents were made at Beamline X23A2 of the National Synchrotron Light Source, Brookhaven National Laboratory. Foil and powder spectra were collected in transmission mode, while the spectra from compacted samples were acquired using electron detection in a He gas detector. Reductions in amplitude similar to those seen previously were observed for the compacted nanocrystalline samples, but, surprisingly, an even larger amplitude reduction was seen in uncompacted powder. A comparison of the data for the three types of samples is seen in Fig. 4. The fact that larger reductions in amplitude are seen for nanocrystalline powder, in which the grain boundary volume is negligible, suggests that large-magnitude, disordered boundary relaxations are not responsible for the observed behavior, in contrast to the conclusion of [2]. A detailed analysis of the data aimed at understanding the role of several effects such as surface oxidation, particle size, vacancies and pores on EXAFS amplitudes is in progress. Comparisons of possible structures derived by molecular dynamics calculations as well as from simple models are being made. Methods have also been developed to extract grain radii from EXAFS amplitudes of different atomic shells. These techniques are expected to prove useful in making connections between diffraction and EXAFS results.

\section{Acknowledgements}

Use of the facilities at both HASYLAB and NSLS are gratefully acknowledged. J. E. was supported by the U. S. Dept. of Energy, BES-DMS, under Contract W-31-109-Eng-38. M. F. wishes to thank the American-German Fulbright Commission for providing financial support. Additional funding (M. F., M. M. and G. W.) was provided by the German Federal Ministry for Research and Technology (BFMT) under Contracts 523-4003-03M23 and 03PE1LMU/2.

\section{Beferences}

1) X. Zhu, R. Birringer, U. Herr and H. Gleiter, Phys. Rev. B 35, 9085 (1987).

2) T. Haubold, R. Birringer, B. Lengeler and H. Gleiter, Jn. Less-Comm. Metals 145, 557 (1988).

3) G. J. Thomas, R. W. Siegel and J. A. Eastman, Scripta Metall. et Mater. 24, 201 (1990).

4) M. R. Fitzsimmons, J. A. Eastman, M. Müller-Stach and G. Wallner, submitted to Phys. Rev. B.

5) W. T. Elam, J. A. Eastman, K. H. Kim, E. F. Skelton, J. Lupo and M. J. Sabochick, in preparation.

6) J.A. Eastman and L. J. Thompson, in preparation.

7) K. Kimoto, Y. Kamilya, M. Nonoyama and R. Uyeda, Jpn. J. Appl. Phys. 2, 702 (1963).

8) H. Gleiter, in Deformation of Polycrystals: Muchanisms and Microstructures, N. Hansen et al., eds., Risø National Laboratory, Roskilde (1981).

9) From the 69th (1988-1989) Edition of the CRC Handbook of Chemistry and Physics.

10) T. Mütschele and R. Kirchheim, Scripta Metall. 21, 1101 (1987).

11) F. A. Lewis, The Palladlum Hydrogen System, Academic Press, London (1967).

12) J. W. Simons and T. B. Flanagan, Jn. Phys. Chem. 69, no. 11, 3773 (1965). 

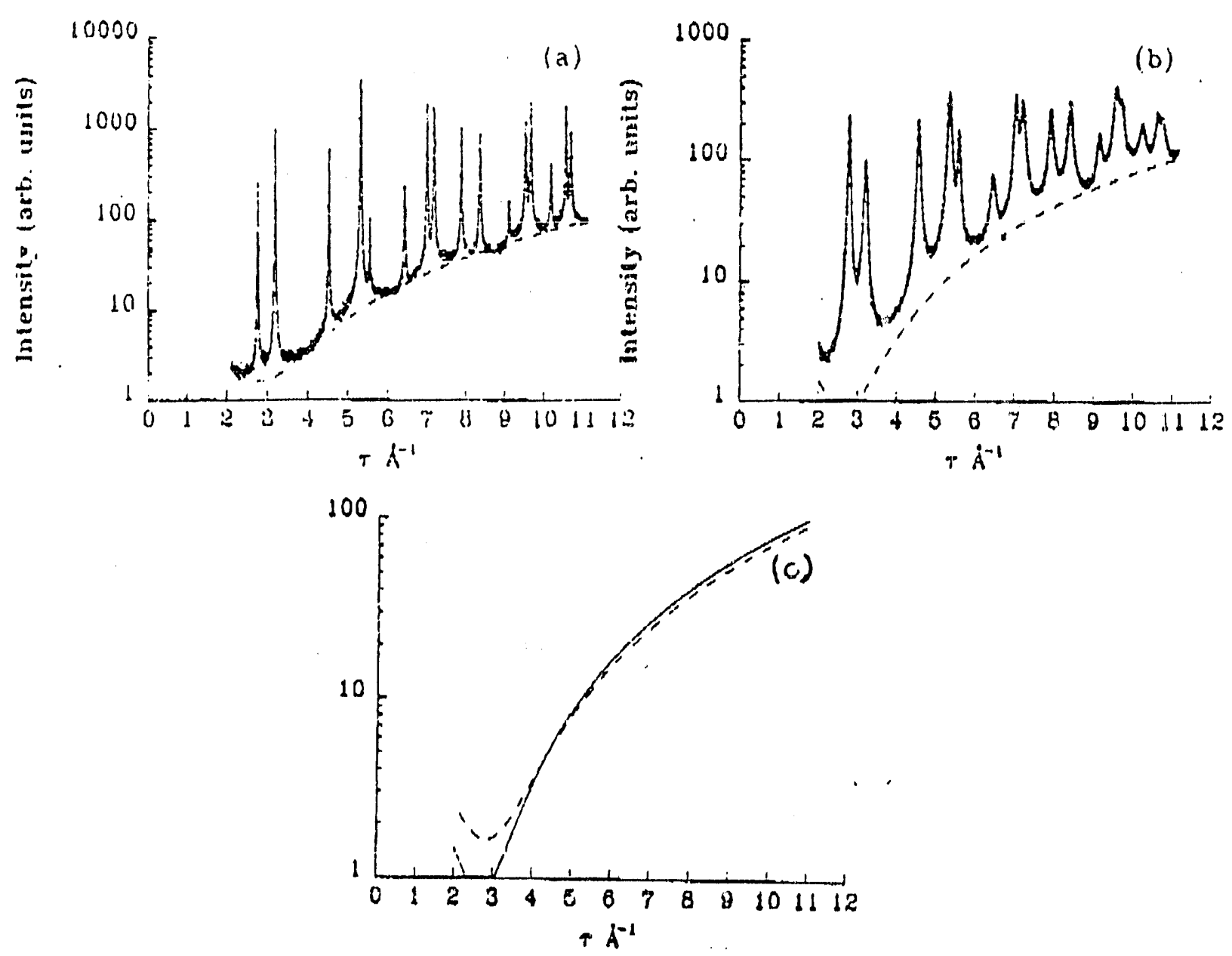

Fig. 1 Corrected intensity data from (a) coarse-grained and (b) nanocrystalline Pd plotted oin a logarithmic scale versus scattering vector magnitude, $\tau=4 \pi \sin \theta / \lambda$. Dashed lines indicate the intensity not accounted for by the Lorentzianshaped Bragg reflections. (c) Quadratic polynomial representation of the background intensity from coarse-grained (dashed line) and nanocrystalline Pd (solid line).

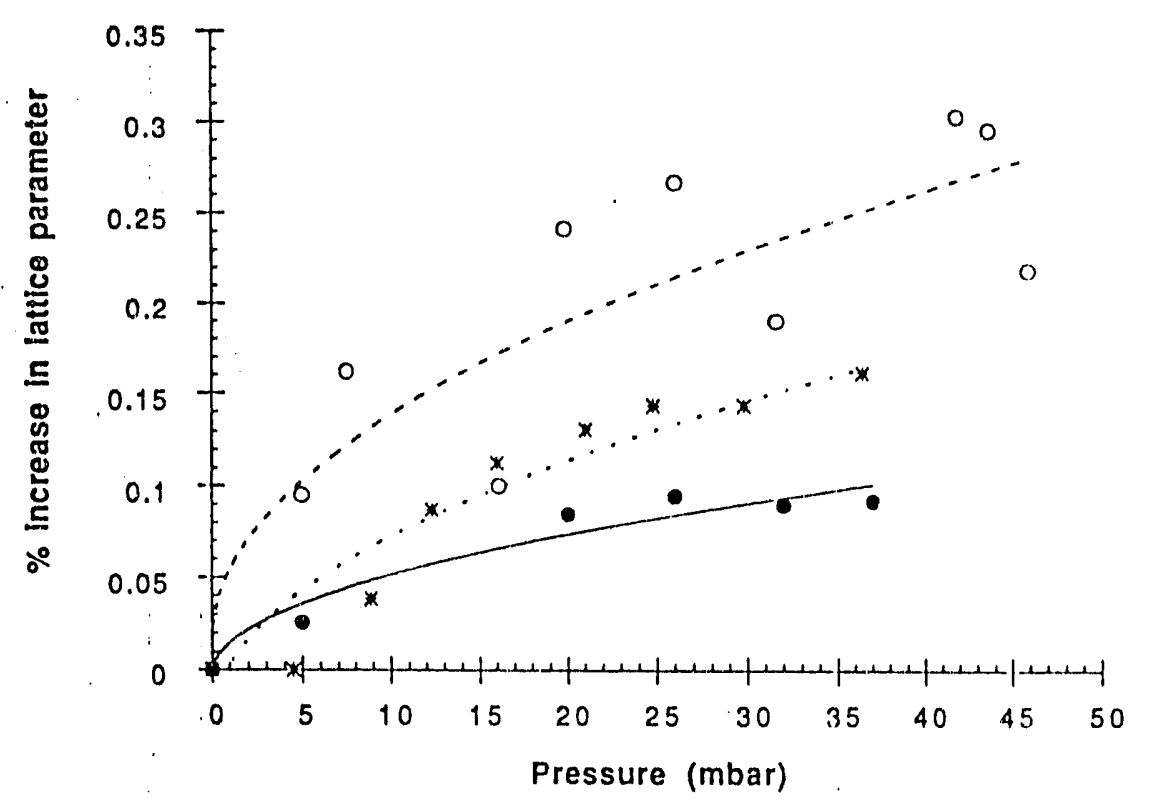

Fig. 2 Change in Pd lattice parameter versus hydrogen pressure for $10 \mathrm{~nm}$ (open circles), $50 \mathrm{~nm}$ (stars) and coarsegrained Pd (closed circles). 

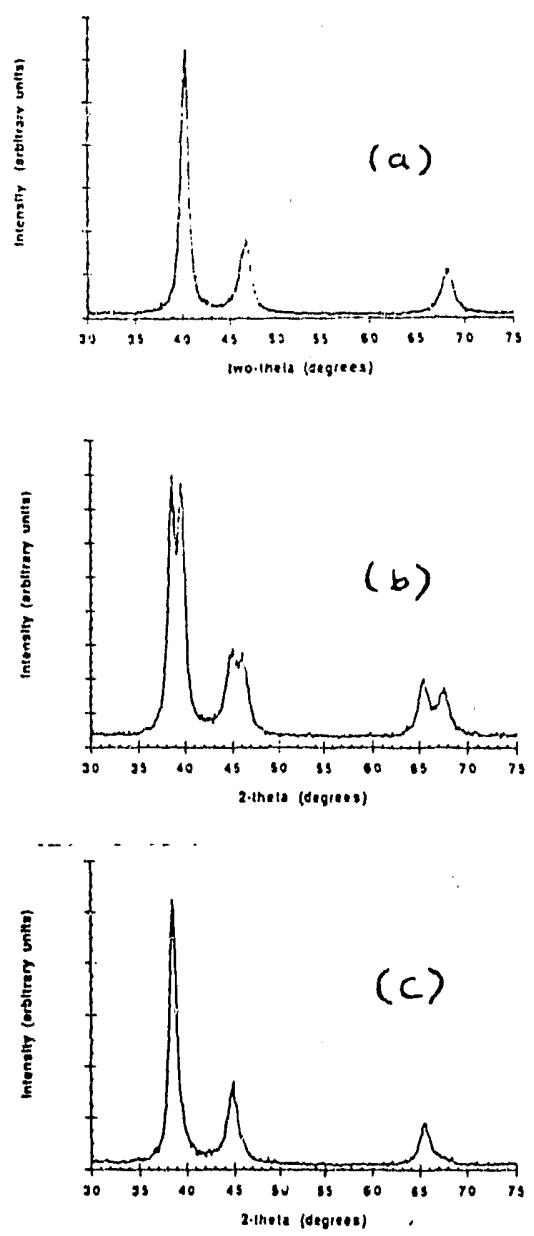

Fig. 3 Intensity data for $10 \mathrm{~nm}$ grain-sized Pd prior to $\mathrm{H}_{2}$ exposure $(\mathrm{a})$, in the mixed-phase $(\mathrm{Pd}+\mathrm{PdH})$ regime (b) and following complete transformation to the hydride phase. The hydrogen pressure was $5.5 \mathrm{kPa}$.

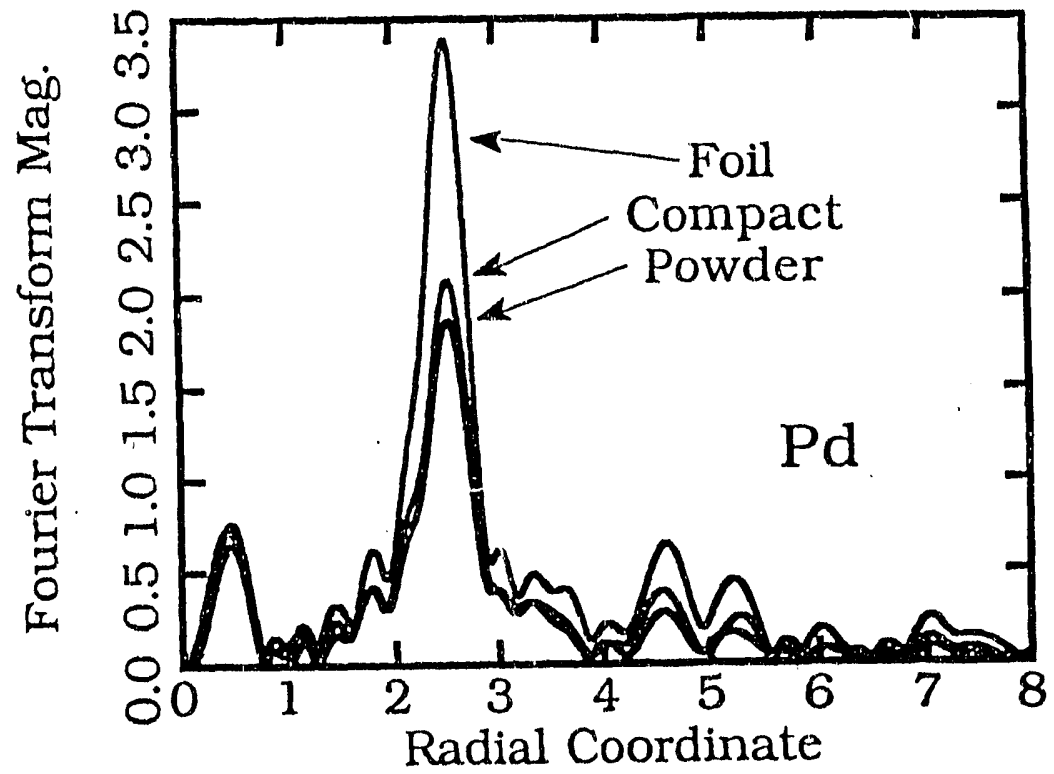

Fig. 4 Fourier transform of the $k^{2}$ weighted EXAFS above the Pd edge for a coarse-grained Pd foil sample, for a compacted nanocrystalline sample and for nanocrystalline powder. The radial coordinate is not corrected for atomic phase shifts (about $0.4 \AA$ ). 

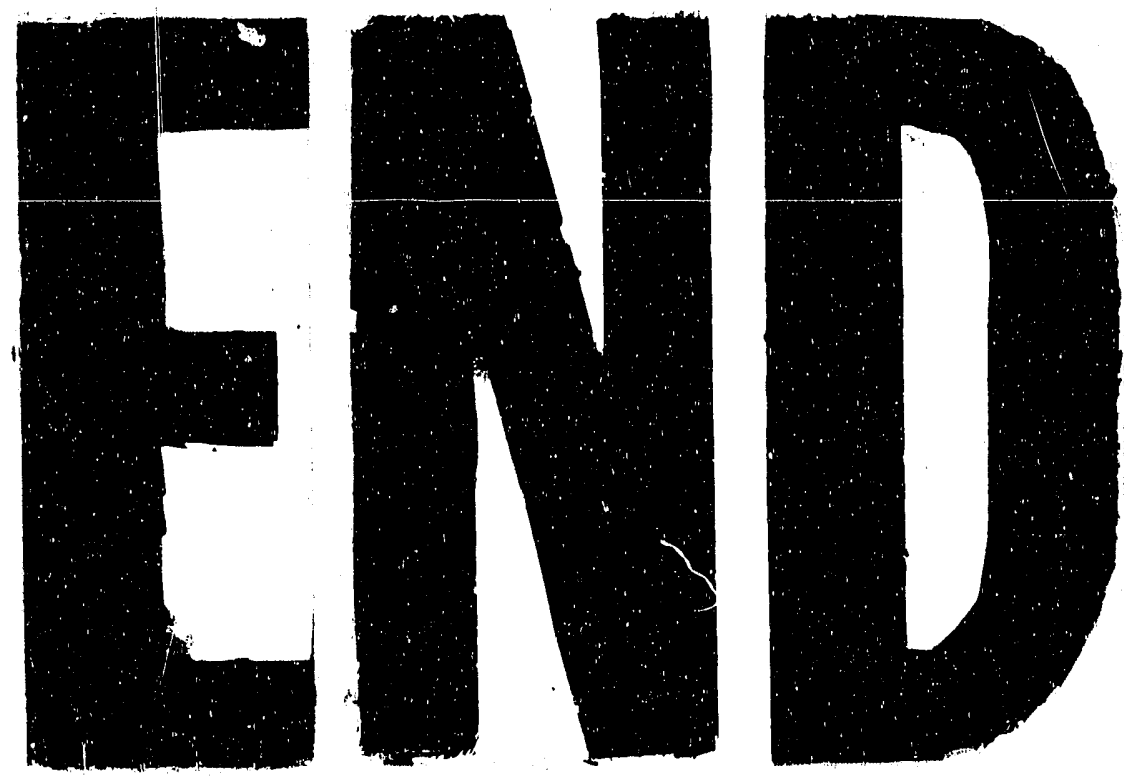

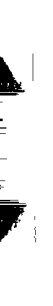

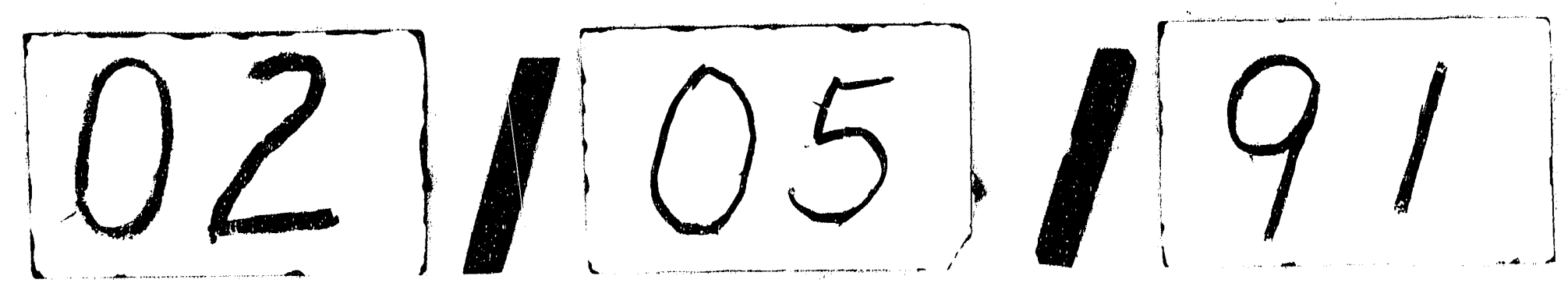


